\title{
Analyzing The Concept Of Graded K-Preference Integration Representation Method
}

\author{
Anant Tiwari ${ }^{1}$, Dr. Amit Kumar Vats ${ }^{2}$ \\ ${ }^{1}$ Research Scholar, School of Applied and Social Sciences, Singhania University, Pacheri Bari, Jhunjhunu, \\ Rajasthan, India \\ ${ }^{2}$ Assistant Professor, School of Applied \& Social Sciences, Singhania University, Pacheri Bari, Jhunjhunu, \\ Rajasthan, India
}

Article History: Received: 11 January 2021; Accepted: 27 February 2021; Published online: 5 April 2021

\begin{abstract}
Generally, the fuzzy set concept could be used to deal with the problems with the qualities of ambiguity as well as vagueness. In the decision making process, the reference comparisons for criteria \& options tend to be more appropriate to make use of the linguistic variables rather than crisp values in some instances. Meanwhile, the GMIR technique is utilized for the constrained trouble construction to derive the weights of options \& criteria, which accomplishes the extension of fuzzy environment. Here in this paper we will study about some basic terms related to K-preference Graded Integration method. We will discuss the fuzzy inventory models under decision maker's preference (k-preference), and find the optimal solutions of these models, the optimal crisp order quantity or the optimal fuzzy order quantity.
\end{abstract}

Keywords: fuzzy, set, theory,models,k-preference, models, etc.

\section{INTRODUCTION}

As a great deal of money is occupied to the inventories coupled with the increased carrying expenses of theirs, the pharmaceutical companies can't manage to have some money tied up in extra inventories. Just about any substantial buy in pharmaceutical inventories might prove to become a major drag on the profitable functioning of a healthcare business. As a result there's a necessity to cope with pharmaceutical inventories far more productively to release the substantial quantity of capital interested in the pharmaceutical materials. Inventory management is actually the supervision of supply, accessibility as well as storage of products to be able to make sure an ample source with no too much oversupply. It is able to additionally be referred as inner command of an accounting process or maybe system regarded as promoting very good business or maybe guarantees the achievement of a policy or perhaps safeguards assets or even stays away from error and fraud, etc. For fiscal region, the inventory management issue, which plan to reduce overhead price without harming revenue. In the area of loss anticipation, methods designed to introduce specialized barriers to shoplifting. Additionally it answers the following simple questions of any supply chain: (1) what to order? (2)When to order? (3) Where to order? (4) Just how much to hold in stock? so as to boost finances. To manage inventories correctly, one has to take into account all cost components which are linked with the inventories. However, there are very few some price essentials, which do impact price of inventory. The entire price of holding inventory is known as Carrying Inventory. This have warehousing expenses including hire fee, salaries and utilities, financial costs including opportunity cost, and inventory costs related to perishability, pilferage, assurance and minimization. Buying price is actually expense of ordering raw materials for pharmaceutical generation purposes. These include cost of putting a buy order, expense of check up of received batches, certification expenses, etc.Chen and Hsieh introduced the Graded k preference Integration Representation method of generalized fuzzy number depending on the essential worth of graded k preference $h$ levels of generalized fuzzy selection.

Till today there's no fuzzy inventory design utilizing k-preference of the pentagonal fuzzy number To ensure that in this particular paper, the economic order amount inventory version with shortage utilizing k preference of the pentagonal fuzzy number continues to be viewed in a fuzzy environment The fuzzy holding price, buying price as well as shortage price have been represented by the pentagonal fuzzy number. The unit is actually defuzzified by k- preference Graded mean Integration technique.

\section{FUZZY NUMBERS AND GRADED MEAN INTEGRATION REPRESENTATION TECHNIQUE}

A fuzzy subset of the actual line $\mathrm{R}$, whose membership functionality $M_{\mathrm{f}}$ satisfies the next situation, is actually a generalized fuzzy number $\tilde{A}$.

i. $\quad M_{\mathrm{f}}$ is a continuous mapping from $\mathrm{R}$ to the closed interval $[0,1]$,

ii. $\quad M_{\mathrm{f}}=0, a_{1}-\infty<\mathrm{x} \leq$

iii. $\quad M_{\mathrm{f}}=\mathrm{L}(\mathrm{x})$ is strictly increasing on $\left[a_{1}, a_{2}\right]$

iv. $\quad M_{\mathrm{f}}=W_{\mathrm{A}}, a_{2} \leq \mathrm{x} \leq a_{3}$

v. $\quad \mathrm{Mf}=\mathrm{R}(\mathrm{x})$ is strictly decreasing on $\left[a_{3}, a_{4}\right.$, ] 
vi. $\quad M_{\mathrm{f}}=0, a_{4} \leq \mathrm{x} \leq \infty$

Where $0<W_{\mathrm{A}} \leq 1$ and $a_{1}, a_{2}, a_{3}$ and $a_{4}$ are real numbers. Moreover, this kind of generalized fuzzy number is denoted as $\tilde{A}=\left(a_{1}, a_{2}, a_{3} a_{4} W_{A}\right)_{\mathrm{LR}}$ when $W_{A}=1$, it can be simplified as $\tilde{A}=\left(a_{1}, a_{2}, a_{3} a_{4} W_{A}\right)_{\mathrm{LR}}$

Graded Mean Integration Representation Technique

Defuzzification of $\tilde{A}$ can be done by Graded Mean Integration Representation Method. If $\tilde{A}$ is a triangular fuzzy number and is entirely determined by $\left(a_{1}, a_{2}, a_{3}\right)$ then defuzzified value is defined as

$$
G(A)=\frac{1}{2} \frac{\int_{0}^{1} h\left[a_{1}+h\left(a_{2}-a_{1}\right)+a_{3}-h\left(a_{3}-a_{2}\right) d h\right.}{\int_{0}^{1} h d h}=\frac{a_{1}+4 a_{2}+a_{3}}{6}
$$

\section{THE GRADED MEAN INTEGRATION REPRESENTATION OF L-R TYPE FUZZY NUMBERS}

In general, a generalized L-R type fuzzy number A can be described as any fuzzy subset of the real line R whose membership function [[mu].sub.A] satisfies the following conditions.

(1) $[[\mathrm{mu}]$. sub.A] is a continuous mapping from $\mathrm{R}$ to the closed interval $[0,1]$,

(2) $[[\mathrm{mu}] . \operatorname{sub} \cdot \mathrm{A}](\mathrm{x})=0$, - [infinity] $<\mathrm{x}$ [less than or equal to] $\mathrm{c}$,

(3) $[[\mathrm{mu}] . \operatorname{sub} . \mathrm{A}](\mathrm{x})=\mathrm{L}(\mathrm{x})$ is strictly increasing on $[\mathrm{c}, \mathrm{a}]$,

(4) $[[\mathrm{mu}] . \operatorname{sub} . \mathrm{A}](\mathrm{x})=\mathrm{w}$, a [less than or equal to] $\mathrm{x}$ [less than or equal to] $\mathrm{b}$,

(5) $[[\mathrm{mu}] . \operatorname{sub} . \mathrm{A}](\mathrm{x})=\mathrm{R}(\mathrm{x})$ is strictly decreasing on $[\mathrm{b}, \mathrm{d}]$,

(6) $[[\mathrm{mu}] \cdot \operatorname{sub} \cdot \mathrm{A}](\mathrm{x})=0, \mathrm{~d}$ [less than or equal to] $\mathrm{x}<$ [infinity],

where $0<w[$ less than or equal to]1, and a, b, c, d are real numbers. This generalized L-R type fuzzy number is denoted as $\mathrm{A}=(\mathrm{c}, \mathrm{a}, \mathrm{b}, \mathrm{d}$; w)[.sub.LR]. When $\mathrm{w}=1$, we denote $\mathrm{A}=$ (c, a, b, d)[.sub.LR]. For example, when one says that something is "around 1000" with $90 \%$ of confidence, he can give $\quad w=0.9$. Let [L.sup.-1] and [R.sup.-1] be the inverse functions of the functions $\mathrm{L}$ and R, respectively. The graded mean $\mathrm{h}-$ level value of generalized L-R type fuzzy number $A=(c, a, b, d ; w)[. s u b . L R]$ is $h[[L . s u p .-1](h)+[R . s u p .-1](h)] /$. Today, we determine the representation of a generalized L R sort fuzzy number depending on the integration valuation of graded mean $\mathrm{h}$ levels as follow.

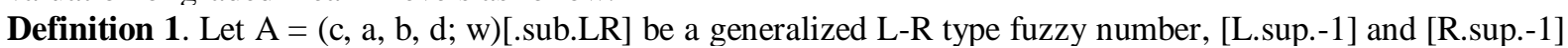
be the inverse functions of the functions $\mathrm{L}$ and $\mathrm{R}$ respectively. Then the Graded Mean Integration Representation (GMIR) of $\mathrm{A}$ is,

$\mathrm{P}(\mathrm{A})=\quad[[$ integral].sub.0.sup.w $] \quad \mathrm{h} \quad([$ lambda $] \quad[$ L.sup.-1](h) $+\quad(1 \quad-\quad[$ lambda $])[$ R.sup.-1](h))dh/ [[integral].sub.0.sup.w] h dh, (1)

where $\mathrm{h}$ is between 0 and $\mathrm{w}, 0<\mathrm{w}$ [less than or equal to] 1, 0 [less than or equal to] [lambda] [less than or equal to] 1. We call $\mathrm{P}(\mathrm{A})$ as graded [lambda]-preference integration representation of fuzzy number $\mathrm{A}$. Remark 1. When [lambda] = 1/2 in Definition 1, we call P(A) a GMIR of A. The value of [lambda] depends on the preference of the decision maker. Usually we choose [lambda] $=1 / 2$, since it does not bias to left or right. Remark 2. When [L.sup.-1](h) or [R.sup.-1](h) does not exist, or [[integral].sub.0.sup.w] h[[[L.sup.-1](h) + [R.sup.-1] (h)]/2] dh cannot integrate, we can divide $[0, \mathrm{w}]$ into $\mathrm{n}$ equal intervals, and let $\mathrm{P}(\mathrm{A})=[[$ n.summation over $(\mathrm{i}=1)] \mathrm{w}(\mathrm{i} / \mathrm{n})([$ L.sup. -1$](\mathrm{w}[\mathrm{i} / \mathrm{n}])+[$ R.sup. -1$](\mathrm{w}[\mathrm{i} / \mathrm{n}])) / 2] /[\mathrm{n}$.summation over $(\mathrm{i}=1)]$ $\mathrm{w}(\mathrm{i} / \mathrm{n})$.

Remark 3. From definition of generalized L-R type fuzzy number in Definition 1, we have -[infinity] $<\mathrm{c}$ [less than or equal to] a [less than or equal to] $\mathrm{b}$ [less than or equal to] $\mathrm{d}<$ [infinity].

\section{FORMULATION OF THE CRISP MODEL}

To infer the inventory cost function for the primary booking time frame $\mathrm{T}$, we partition the time stretch $[0, T]$ into two sections: $\left[0, t_{1}\right]$ and $\left[t_{1}, T\right]$. The creation begins at time $t=0$ and stops at timet $=t_{1}$. In this way, stock develops during the time frame $\left[0, t_{1}\right]$ and decays during the time frame $\left[t_{1}, T\right]$. In the event that $I_{1}(t)$ and $I_{2}(t)$ signify, individually, the inventory levels whenever during the time-frames $\left[0, t_{1}\right] \operatorname{and}\left[t_{1}, T\right]$, at that point the differential conditions addressing the inventory status are given by

$\frac{d I_{1}(t)}{d t}=p-d-\frac{E(N)}{t_{1}}, 0 \leq t \leq t_{1}$ With $I_{1}(0)=0$,

$\frac{d I_{2}(t)}{d t}=-d-\frac{E(N)}{t_{1}}, t_{1} \leq t \leq T$ With $I_{2}(0)=0$,

Where $E(N)$, the normal number of flawed things created during the creation run is determined as given beneath: On the off chance that the interaction shifts at timet $\left(0 \leq t \leq t_{1}\right)$ at that point the all out number of things created after cycle move is $p\left(t_{1}-t\right)$. Consequently, the normal number of inadequate things created during the creation run is

$E(N)=\int_{0}^{\infty} \gamma p\left(t_{1}-t\right) f_{X}(t) d t=\int_{0}^{t_{1}} \int_{0}^{\infty} \gamma p\left(t_{1}-t\right) \lambda e^{-\lambda t} d t=\frac{\gamma p}{\lambda}\left[e^{-\lambda t_{1}}+\lambda t_{1}-1\right](4)$ 
Utilizing (4) in (2) and afterward addressing the differential conditions (2) and (3), we acquire

$I_{1}(t)=(p-d) t-\frac{\gamma p}{\lambda t_{1}}\left[e^{-\lambda t_{1}}+\lambda t_{1}-1\right] t, 0 \leq t \leq t_{1}(5)$

$I_{2}(t)=d(T-t), t_{1} \leq t \leq T \quad(6)$

In this way, inventory holding cost is as per the following,

$$
h\left[\int_{0}^{t_{1}} I_{1}(t) d t+\int_{t_{1}}^{T} I_{2}(t) d t\right]=\frac{h}{2 \lambda}\left[\lambda p t_{1}^{2}+\lambda d T^{2}-2 \lambda d t_{1} T-\gamma p t_{1}\left(e^{-\lambda t_{1}}+\lambda t_{1}-1\right)\right],
$$

Furthermore, defective thing cost is $c E(N)=\frac{c \gamma p}{\lambda}\left(e^{-\lambda t_{1}}+\lambda t_{1}-1\right)$.

The absolute cost per unit time $(W)$ which is the time normal of the amount of set up cost, holding cost and deficient thing cost is given by

$W\left(t_{1}\right)=\frac{K}{T}+\frac{p}{2 \lambda T}\left[h\left\{\lambda p t_{1}^{2}+\lambda d T\left(T-2 t_{1}\right)\right\}+\gamma p\left(2 c-h t_{1}\right)\left(e^{-\lambda t_{1}}+\lambda t_{1}-1\right)\right]$

Since $I_{1}\left(t_{1}\right)=I_{2}\left(t_{1}\right)$, in this manner, we have $T=\frac{1}{d \lambda}\left\{\lambda p t_{1}-\gamma p\left(e^{-\lambda t_{1}}+\lambda t_{1}-1\right)\right\}$

Revamping the terms, Eq. (7) can be reworked as

$$
W\left(t_{1}\right)=\frac{K}{T}+\frac{p}{2 \lambda T}\left[\lambda h t_{1}^{2}+\gamma\left(2 c-h t_{1}\right)\left(e^{-\lambda t_{1}}+\lambda t_{1}-1\right)\right]+\frac{d h}{2}\left(T-2 t_{1}\right)
$$

Presently subbing $p=\frac{1}{\beta} d$ and $T=\frac{1}{d \lambda}\left\{\lambda p t_{1}-\gamma p\left(e^{-\lambda t_{1}}+\lambda t_{1}-1\right)\right\}$ in the above condition, we get

$$
\begin{aligned}
W\left(t_{1}\right)=\frac{K \lambda \beta}{\lambda t_{1}-\gamma\left(e^{-\lambda t_{1}}+\lambda t_{1}-1\right)} \\
+\frac{\lambda d p}{2 \lambda p\left\{\lambda t_{1}-\gamma\left(e^{-\lambda t_{1}}+\lambda t_{1}-1\right)\right\}}\left[-\lambda t_{1}\left(2 c-h t_{1}\right)+2 c \lambda t_{1}+\gamma\left(2 c-h t_{1}\right)\left(e^{-\lambda t_{1}}+\lambda t_{1}\right.\right. \\
-1)]+\frac{d h}{2 \lambda \beta}\left\{\lambda t_{1}-\gamma\left(e^{-\lambda t_{1}}+\lambda t_{1}-1\right)\right\}-d h t_{1},
\end{aligned}
$$

This after improvement gives

$W\left(t_{1}\right)=-\frac{d}{2}\left(2 c-h t_{1}\right)+\frac{\lambda\left(c d t_{1}+K \beta\right)}{\lambda t_{1}-\gamma\left(e^{\left.-\lambda t_{1}+\lambda t_{1}-1\right)}\right.}+\frac{d h}{2 \lambda \beta}\left\{\lambda t_{1}-\gamma\left(e^{-\lambda t_{1}}+\lambda t_{1}-1\right)\right\} .(8)$

The target of this crisp model is to figure out the ideal creation time $t_{1}^{*}$ which limits the cost per unit time W.

\section{METHODS OF GRADED MEAN INTEGRATION REPRESENTATION}

The evaluated mean integration portrayal of $\tilde{A}$ is $p(\tilde{A})$ where

$$
p(\tilde{A})=\frac{\frac{1}{2} \int_{0}^{1} h\left[L^{-1}(h)+R^{-1}(h)\right] d h}{\int_{0}^{1} h d h} \text { with } 0<h \leq 1
$$

By the above equation, the evaluated mean integration portrayal of three-sided fuzzy number $\tilde{A}=\left(a_{1}, a_{2}, a_{3}\right)$, trapezoidal fuzzy number $\tilde{B}=\left(b_{1}, b_{2}, b_{3}, b_{4}\right)$ are given by

\subsection{Median Rule}

$$
\begin{gathered}
p(\tilde{A})=\frac{a_{1}+4 a_{2}+a_{3}}{6} \\
p(\tilde{B})=\frac{b_{1}+2 b_{2}+2 b_{3}+b_{4}}{6}
\end{gathered}
$$

One method of changing a fuzzy set into a real number is described by picking median that separates the zone under the participation work into halves (middle guideline). In the event that $\tilde{A}=\left(a_{1}, a_{2}, a_{3}\right)$ a three-sided fuzzy number, at that point by middle guideline the defuzzified estimation of $\tilde{A}$ is given by $p(\tilde{A})=\frac{a_{1}+2 a_{2}+a_{3}}{4}$

On the off chance that $\widetilde{B}=\left(b_{1}, b_{2}, b_{3}, b_{4}\right)$ is a trapezoidal fuzzy number, at that point by median standard the defuzzified estimation of $\tilde{B}$ is given by $p(\widetilde{B})=\frac{b_{1}+b_{2}+b_{3}+b_{4}}{4}$

\subsection{Centroid Method}

The Center of Gravity strategy (COG) or Centroid technique is the most insignificant weighted normal and has an unmistakable mathematical implying that is the focal point of gravity or focus of mass. From the numerical perspective the COG compares to the normal estimation of probability. It is characterized as

$$
z_{C O G}=\frac{\int_{z} z \mu(z) d z}{\int_{z} \mu(z) d z}
$$

\subsection{Signed Distance Method}

The signed distance presented by Jing-Shig Yao, Kweimei Wu has some comparable properties to the properties initiated by the signed distance in real numbers. For anyaand $0 \epsilon R$, characterize the signed separation from a to 0 as $d_{0}(a, 0)=a$. In the event that $a>0$, the separation from a to 0 is $-a=-d_{0}(a, 0)$. if a $<$. 
From the meaning of signed distance, the signed distance of two end purposes of the $\alpha-\operatorname{cut} B(\alpha)=$ $\left[B_{L}(\alpha), B_{U}(\alpha)\right]$ of $\tilde{B}$ to the birthplace 0 is $d_{0}\left(B_{L}(\alpha), 0\right)=B_{L}(\alpha)$, and $d_{0}\left(B_{U}(\alpha), 0\right)=B_{U}(\alpha)$, individually. Their normal $\frac{B_{L}(\alpha)+B_{U}(\alpha)}{2}$ is taken as the signed distance of $\alpha-\operatorname{cut}\left[B_{L}(\alpha), B_{U}(\alpha)\right]$ to 0 . That is signed distance of span $\left[B_{L}(\alpha), B_{U}(\alpha)\right]$ to 0 is characterized as

$$
\left.d_{0}\left[B_{L}(\alpha), B_{U}(\alpha)\right], 0\right)=\frac{1}{2}\left[d_{0}\left(B_{L}(\alpha), 0\right)+d_{0}\left(B_{U}(\alpha), 0\right)\right]=\frac{B_{L}(\alpha)+B_{U}(\alpha)}{2}
$$

The signed distance of $\mathrm{B}$ to 0 is characterized as

$$
d(\widetilde{B}, 0)=\frac{1}{2} \int_{0}^{1}\left(\left[\left(B_{L}(\alpha)+B_{U}(\alpha)\right)\right]\right)
$$

For the triangular number $\tilde{A}=\left(a_{1}, a_{2}, a_{3}\right)$ the $\alpha$ - cut of $\tilde{A}$ is $A(\alpha)=\left[A_{L}(\alpha), A_{U}(\alpha)\right], \alpha \in[0,1]$ where $A_{L}(\alpha)=$ $a_{1}+\left(a_{2}-a_{1}\right) \alpha$ and $A_{U}(\alpha)=a_{1}+\left(a_{2}-a_{1}\right) \alpha$.

The signed distance of $\tilde{A}$ to 0 is $d(\tilde{A}, 0)=\frac{1}{4}\left(a_{1}+2 a_{2}+a_{3}\right)$

For the trapezoidal number $\tilde{B}=\left(b_{1}, b_{2}, b_{3}, b_{4}\right)$ the $\alpha$-cut of $\tilde{B}$ is $B(\alpha)=\left[B_{L}(\alpha), B_{U}(\alpha)\right], \alpha \in[0,1]$ where $B_{L}(\alpha)=b_{1}+\left(b_{2}-b_{1}\right) \alpha$ and $B_{U}(\alpha)=b_{1}+\left(b_{2}-b_{1}\right) \alpha$.

The signed distance of $\tilde{B}$ to 0 is $d(\widetilde{B}, 0)=\frac{1}{4}\left[b_{1}+b_{2}+b_{3}+b_{4}\right]$

\section{CONCLUSION}

To sum up all that's been claimed thus far, we derive a number of attributes of the representation of fuzzy amounts by utilizing the GMIR method below fuzzy arithmetical activities with extension concept. These attributes are able to help us to streamline the calculation of representation of kth order plane curve fuzzy numbers, the multiplication of 2 or perhaps 3 fuzzy numbers as well as the linear mix of the multiplication of fuzzy figures. When working with these formulas, we do not need to go through the businesses of the membership feature to receive the representation of fuzzy figures. What we want allow me to share the vertexes of the initial club membership functions of the fuzzy figures only.

\section{REFERENCES}

1. Lic C.J., and Wen U.P., (2004), a Labeling Algorithm for the Fuzzy Assignment Problem, Fuzzy Sets and Systems. 142: $373-391$.

2. Ridwan M., (2004), Fuzzy preference Based Traffic Assignment Problem, Transportation Res. Part C, 12:209-233.

3. J. J. Buckley, A fast method of ranking alternatives using fuzzy numbers, Fuzzy sets and Systems, 30 (1989) 337-338.

4. L. Campos and J. L. Verdegay, Linear programming problems and ranking of fuzzy numbers, Fuzzy sets and Systems 32 (1989) 1-11.

5. P. T. Chang and E. S. Lee, Fuzzy arithmetics and comparison of fuzzy numbers, Fuzzy optimization: Resent advances (M. Delgado, J. Kacprzyk, J.-L. Verdegay, M. A. Vila), Physica-Verlag, Heidelberg, Germany, 69-81.

6. S. Heilpern, Representation and application of fuzzy numbers, Fuzzy Sets and Systems 91 (1997)259268.

7. R. Jain, Decision-making in the presence of fuzzy variables, IEEE Trans., Systems Man and Cybern. 6 (1976), 698-703.

8. R. Jain, A procedure for mutli-aspect decision making using fuzzy sets, Int. J. Systems Science 8 (1977), $1-7$.

9. Kaufmann and M. M. Gupta, Introduction to Fuzzy Arithmetic Theory and Applications, Van Nostrand Reinhold, 1991.

10. Shan-Huo Chen and Chin Hsun Hseih, (2000), Graded Mean Integration Representation of Generalized Fuzzy Number journal of the chunese Fuzzy System Association, Taiwan, 5(2): 1-7.

11. Bogomolnaia A., and MoulinA.,(2001), A New solution to the Random Assignment Problem, Journal of Economic Theory, 100: 295-328.

12. Bogomolnaia A., and Moulin H., (2002), A Simple Random Assignment problem with a Unique Solution, Economic Theory, 19: 623-636.

13. Belacela N., and Boulasselb M.R., (2001), Multicriteria Fuzzy Assignment Method: A Useful Tool to Assist Medical Diagnosis, Artificial Intelligence in Medicine, 21: 201 - 207. 